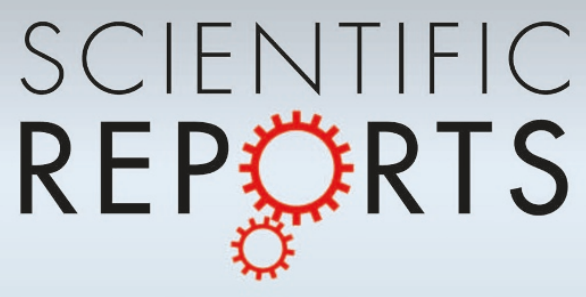

OPEN

SUBJECT AREAS:

HUMAN BEHAVIOUR

AUTISM SPECTRUM DISORDERS

Received

4 November 2013

Accepted

20 February 2014

Published

7 March 2014

Correspondence and requests for materials should be addressed to L.Q.Z. (zhulq@psych. ac.cn)

\section{The relationship between moral judgment and cooperation in children with high-functioning autism}

\author{
Jing Li' ${ }^{1}$ Liqi Zhu' \& Michaela Gummerum²
}

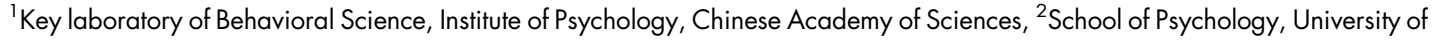 \\ Plymouth, Plymouth, UK.
}

This study investigated moral judgment in children with high-functioning autism and their cooperation in prisoner's dilemma game with partners of different moralities. Thirty-eight 6- to 12-year-old high-functioning autistic (HFA) children and 31 typically developing (TD) children were recruited. Children were asked to judge story protagonists' morality. After making this moral judgment correctly, they were asked to play with the morally nice and the morally naughty child in a repeated prisoner's dilemma game. Results showed that both HFA and TD children made correct moral judgments, and that HFA children might even have more rigid criteria for what constitutes morally naughty acts. HFA children's cooperation did not differ depending on the morality of the interaction partner, while TD children showed higher cooperation when interacting with the morally nice than the morally naughty child did. Thus, partner's morality did influence TD children's but not HFA children's subsequent cooperation.

utism is a neurobiological disorder that is characterized by deficits in social interaction, impairments in communication and language, restricted/repetitive behaviors and/or interests (DSM-IV). Lack of respon-

sivity to social emotional cues or reciprocal engagement is commonly seen as a main characteristic of individuals with autism spectrum disorder (ASD) ${ }^{1}$. Clinical research argues that children with autism lack empathic behavioral responses ${ }^{2,3}$. Studies have shown that children with autism have social-emotional deficits and are inclined to use cognitive strategies to decode emotions in others ${ }^{4}$. Even individuals with high functioning autism (HFA)have deficits in the cognitive and affective components of empathy $y^{5}$ HFAs have difficulties with passing false belief tasks, that is in recognizing that another person's belief might be different from their own and from actual reality ${ }^{6}$. HFAs also show great difficulties with recognizing the expressions of emotion in others ${ }^{7}$ and in perceiving behavior as emotionally expressive $e^{8}$. Successfully passing false belief tests and recognizing emotions in others requires cognitive empathy, specifically theory of mind (ToM).

Moral judgment, that is the ability to judge one's own and others' behavior as right or wrong, is considered to be closely related to ToM, as it is necessary to understand others' beliefs, desires, and intentions to make proper moral judgments9. At least by middle childhood, normally developing individuals pay more attention to a person's intention behind an action than to the outcome of the action when they evaluate the moral permissibility of the action. This ability to focus on others' intentions depends on $\mathrm{ToM}^{10}$.

In addition, empathy is typically considered as a necessary component of developing moral agents ${ }^{11}$. Empathy may play at least two roles in the development of moral agency: (i) empathic ability might be helpful to learn important moral lessons; (ii) empathic responses to harm suffered by a victim might be important in shaping moral judgments to wrong-doing ${ }^{12}$. Furthermore, empathic capacity is useful in understanding who is judged to be a victim of moral transgression ${ }^{13}$.

Moral judgment, as an important aspect of social functioning, has rarely been studied in research on autism ${ }^{13}$, even though individuals with ASD have difficulties in processing mental state information (such as belief and intention) and show deficits in their empathic capacities. Findings on whether the moral judgment of individuals with ASD is different from normally developing individuals have been mixed so far. Moran, et al. ${ }^{14}$ found that ASD individuals, who were impaired in social functioning(including empathy),judged accidental and intentional harm as morally similar. This suggests that ASD adults had difficulties in integrating mental state information in their moral judgments. Leslie, et al. ${ }^{13}$ found that autistic children behaved similarly to normally developing children in their basic moral judgment. In their study, children were asked to judge whether the protagonist in moral stories (who either shared with others or hit others to receive a toy) was morally nice or naughty, and 
whether the protagonist should be rewarded or punished. Similar to normally developing children, children with autism performed appropriately to story type, that is they judged naughty acts as naughty and nice acts as nice.

Moreover, the distinction between moral and conventional rules and violations is considered critical in moral development ${ }^{15}$ : Moral transgressions have negative effects on others' welfare and rights and are wrong independent of the rules set by adults or other authorities. Social conventions, in contrast, are context-specific and rather arbitrary agreements that help to coordinate people's activities and interactions in social systems and organizations (e.g., schools, work places, groups) and are upheld by authority or social expectations ${ }^{16}$. Blair ${ }^{17}$ found that, just like normally developing children, children with ASD were able to distinguish between moral and conventional violations, despite their deficit in ToM. However, Feng and $\mathrm{Su}^{18}$ found that Chinese children with autism had difficulties in making moral judgment due to their deficits in ToM, but they performed similar to normally developing children in their conventional judgments. Grant, et al. ${ }^{19}$ found that, similar to control participants, children with autism judged a moral transgression based on its motivation and believed that an action harming another person should be punished more than an action that caused damage to property.

While autistic children's moral judgment might differ in some respects from those of normally developing children, few studies have investigated how their moral judgments affect their own moral behavior. Do children with autism exhibit different moral behavior towards people they judge as morally nice versus those they judge as morally naughty? The current study examined this question in more detail.

Children's abstract moral reasoning about agents in hypothetical stories is closely related to their daily prosocial behavior ${ }^{20,21}$. Furthermore, children's own moral and prosocial actions are affected by the recipient's moral character or his or her previous (moral or immoral) behavior. For example, Olson and Spelke ${ }^{22}$ found that 3.5year-old normally developing children allocated more resources to a doll who was generous towards the participants or a doll who was described as generally generous than to non-generous dolls. Similarly, Kenward and Dahl ${ }^{23}$ showed that 4.5-year-old children distributed more resources to a puppet that had previously helped than a puppet that had previously hindered another puppet. Thus, these studies indicate that judgments of the moral deservingness of others affect the resource allocations of normally developing children. As discussed above, autistic children behaved similar to normally developing children when they make moral judgment about nice/naughty actions and whether to reward/punish those actions ${ }^{13}$.

In this study, we tested the very basic distinction between "nice" and "naughty". Moral judgments are not simply about what is naughty but also about what is nice ${ }^{24}$. We tested children with HFA on both antisocial and prosocial acts to determine whether they could make both kinds of moral judgments correctly compared to typically developing (TD) children. After making moral judgments properly, participants were asked to interact with protagonists, whom they judged as either nice or naughty before, in the prisoner's dilemma game (PDG). While Downs and Smith ${ }^{8}$ found that highfunctioning children with autism show similar cooperative social behavior in the prisoner's dilemma game as TD children, participants with HFA cooperate to a different degree with a human or computer partner ${ }^{25}$. This indicates that the identity of the opponent matters for HFA's cooperative choices ${ }^{25}$. In this study, we bring these two lines of research together to assess whether their judgments about their interaction partner's morality influences cooperation in children with HFA and normally developing children in prisoner's dilemma game.

Based on the findings byLeslie, et al. ${ }^{13}$, we hypothesized that HFA children would correctly judge others as morally nice or naughty in

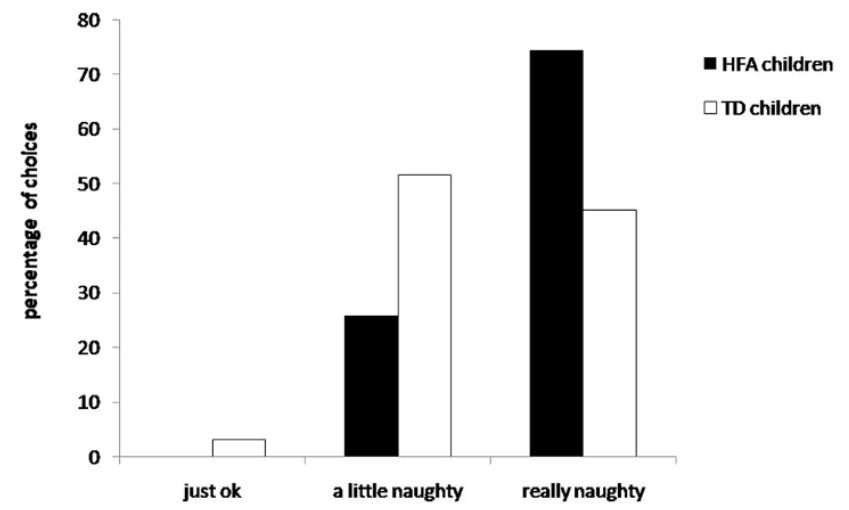

Figure $1 \mid$ described HFA children's and TD children's moral judgment in naughty condition story. Both HFA children and TD children could judge other's morality correctly in naughty condition, and HFA children might even have more rigid criteria for harm to the victim.

the moral stories, similar to typically developing children. However, because of their difficulties with understanding others' intentions, HFA children might exhibit similar cooperative behavior when they were partnered with people they judged as morally nice and naughty. In contrast, in line with earlier research ${ }^{22,23}$, we expected that normally developing children would cooperate more with a partner they evaluated as morally nice than a partner they evaluated as morally naughty.

\section{Results}

Empathy. The Empathy Quotient Child (EQ-C) questionnaire ${ }^{26}$, based on parent report, was adopted to measure all 38 HFA children's and 30 of the 31 TD children's empathic ability. An independent-sample $t$-test showed a significant difference in empathic ability between HFA and TD children, $t(47)=8.01, p=0.00\left(M_{\mathrm{HFA}}=14.50, S D_{\mathrm{HFA}}=5.72\right.$, $\left.M_{\mathrm{TD}}=29.53, S D_{\mathrm{TD}}=8.53\right)$.

Children's moral judgment. Thirty-five of the $38 \mathrm{HFA}$ children and all 31 TD children completed the moral judgment task. In the naughty condition, 35 HFA children chose "naughty" on the good/ naughty question, while $30 \mathrm{TD}$ children chose "naughty", with the remaining 1 TD children choosing "just ok", as shown in Figure 1. An independent sample $t$-test showed that HFA children judged harming others as significantly morally worse than TD children $\operatorname{did}\left(t(57)=2.57, p=0.01<0.05 ; M_{\mathrm{HFA}}=1.74, S D=0.44, M_{\mathrm{TD}}\right.$ $=1.42, S D=0.56)$. Thus, both HFA children and TD children could judge other's morality correctly in naughty condition, and HFA children might even have more rigid criteria for morally judging harming a victim.

In the nice condition, two HFA children chose "just ok" and "a little naughty" respectively. These two HFA children were not asked to continue with the PDG. Children's moral judgment in the nice condition is shown in Figure 2. Both HFA children and TD children could judge others' morality correctly in the nice condition. There was no significant difference in judgment of other's morality between HFA children and TD children in the nice condition $(t(63)=0.25, p$ $\left.=0.80>0.05 ; M_{\mathrm{HFA}}=1.65, S D=0.54, M_{\mathrm{TD}}=1.61, S D=0.56\right)$.

Children's cooperation when interacting with partners of different morality. HFA children and TD children were both asked to play with partners of different morality in the PDG to examine whether they would perform differently when they interacted with morally nice or with morally naughty people. Seven HFA children did not complete the PDG because they could not understand the rules of the game (the two HFA children who did not properly judge the story characters' morality were included in 


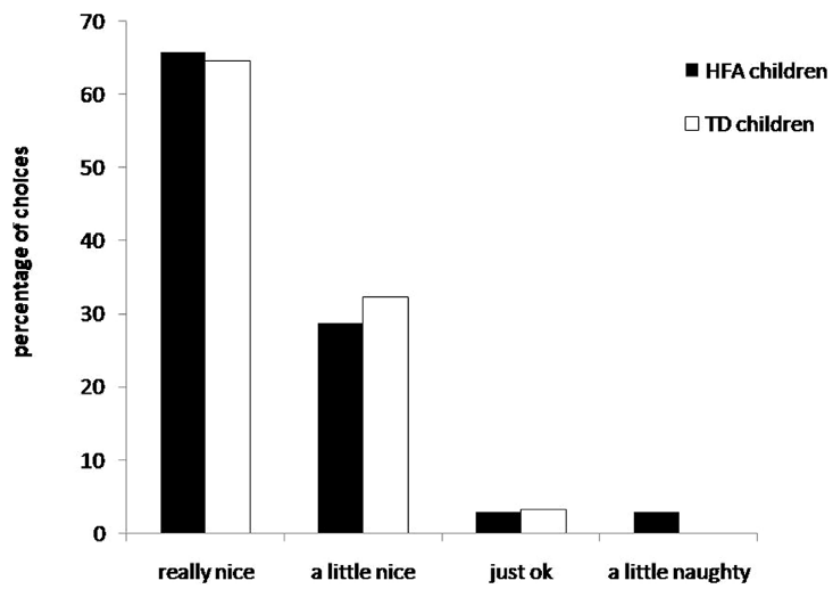

Figure 2 | described HFA children's and TD children's moral judgment in nice condition story. Both HFA children and TD children could also judge other's morality correctly in nice condition. There was no significant difference in judgment of other's nice morality between HFA children and TD children.

these seven HFA children). Accordingly, 31 HFA children ( 25 boys, 6 girls) interacted with morally nice or naughty partners in the PDG. Thirty-one TD children, who were matched in age and gender to HFA children, also completed the PDG. A cooperative response was recorded as 1 point and non-cooperative response was recorded as 0 points. Since ten rounds of PDG were played per interaction partner, scores could range from 0 (no cooperation in all ten games) to 10 (full cooperation in all ten games). HFA and TD children's cooperation when they interacted with partners of different moralities and the random stranger are shown in Table 1.

The difference between children's cooperative response and a random level of cooperation (5) was examined using one-sample $t$-test, shown in Table 1. HFA children cooperated significantly less than the random level when they played with a naughty child, but not different from the random level when they played with the nice child. TD children did not cooperate differently with the random level when they interacted with the naughty child but showed significantly higher than random cooperation when they played with the nice child.

In order to examine the effect of partner's morality on children's cooperation, comparison between their performance when they played with nice/naughty child and performance when they played with the random stranger was tested using a repeated-measures Analysis of Variance (ANOVA). HFA children cooperated similarly with different kinds of partner, $F(2,90)=1.89, p=0.16$. Further post hoc multiple comparison showed that HFA children's cooperation was marginally higher when they were partnered with a nice child than when they were partnered with a naughty child $(p=0.06)$, but their cooperation with a random stranger was not significantly different from cooperating with either a naughty or a nice child. In contrast, TD children showed significantly different cooperative behavior when they were partnered with the three different kinds of players, $F(2,90)=4.13, p=0.02$. Further post hoc multiple comparison showed that TD children's cooperation was significantly higher when they were partnered with a nice child than when they were partnered with a random stranger $(p=0.02)$, or with a naughty child $(p=0.02)$. There was no significant difference in cooperation between playing with a naughty child and playing with a random stranger $(p=1.00)$.

In addition, a paired-sample $t$-test was adopted to compare children's mean payoff after 10 rounds when playing with either a naughty child or nice child. It was found that there was no significant difference in HFA children, $t(30)=-1.60, p=0.12$, whereas, TD children's mean payoff was significantly higher when interacting with a nice child than when interacting with a naughty child, $t(30)$ $=-2.52, p=0.02$.

The percentage of choosing a cooperative responses when HFA and TD children played with the nice child and the naughty child across the 10 rounds of the PDG is shown in Figure 3. A repeatedmeasures ANOVA was conducted to measure children's cooperation when they played with partners of different morality with the between-subject-variable Group (HFA children, TD children), the within-subject variable round, and the covariate control variable IQ. The main effect of Group was not significant when participants played with the naughty child, $F(1,61)=2.68, p=0.11, \eta^{2}=$ 0.04 , while it was significant when participants played with the nice child, $F(1,61)=5.97, p=0.02, \eta^{2}=0.09$. HFA children showed similar cooperation levels as TD children when they played with the naughty child, but showed significantly lower cooperation than TD children when they played with the nice child.

The main effect of round was neither significant in the case of playing with the naughty child $\left(F(9,61)=0.960, p=0.47, \eta^{2}=\right.$ $0.02)$ nor when playing with the nice child $(F(9,61)=1.28, p=0.25$, $\left.\eta^{2}=0.02\right)$. However, further analysis showed that when they played with the naughty child, HFA children performed differently in the 10 rounds of the game, $F(9,30)=2.30, p=0.02<0.05, \eta^{2}=0.07$. Post hoc multiple comparisons showed that HFA children's cooperation was significantly lower in the first and third round than that in the fourth and fifth round, and lower in the third round than that in the eighth round, while higher in the fifth round than that in the ninth and tenth round. TD children did not perform significantly differently across the 10 rounds of the game, $F(9,30)=1.01, p=0.43, \eta^{2}=$ 0.03 . When they played with the nice child, neither the HFA children nor the TD performed significantly differently across the 10 rounds (HFA children: $F(9,30)=1.69, p=0.09, \eta^{2}=0.06$; TD children: $F$ $\left.(9,30)=0.48, p=0.89, \eta^{2}=0.02\right)$. No further significant main or interaction effects emerged.

\section{Discussion}

This study had two main aims: First, to examine whether HFA children could make correct moral judgments, similar to TD children; and second, whether an interaction partner's morality affected cooperation in HFA and TD children. Concerning the first aim, both HFA

Table 1 | Children's cooperative behavior in PDG with different kinds of partner

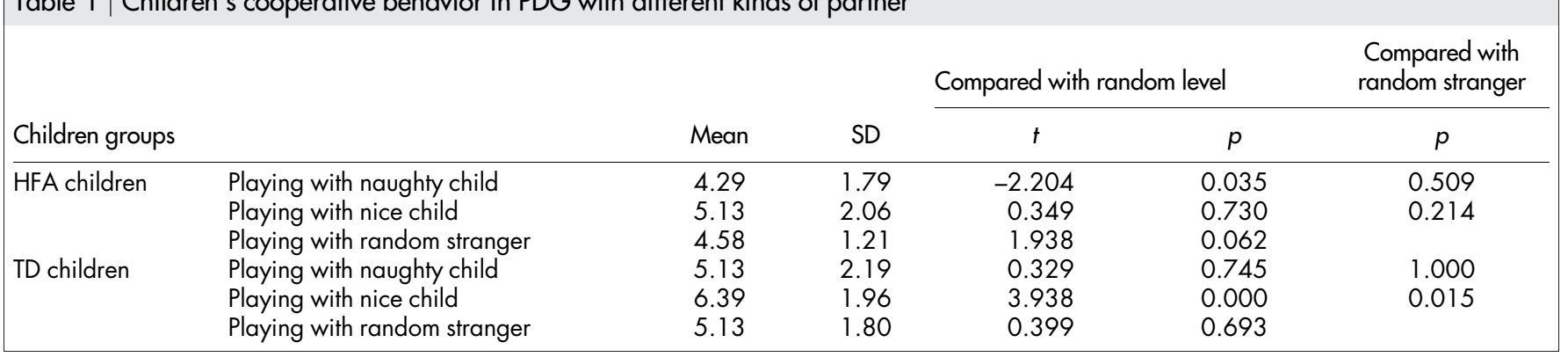




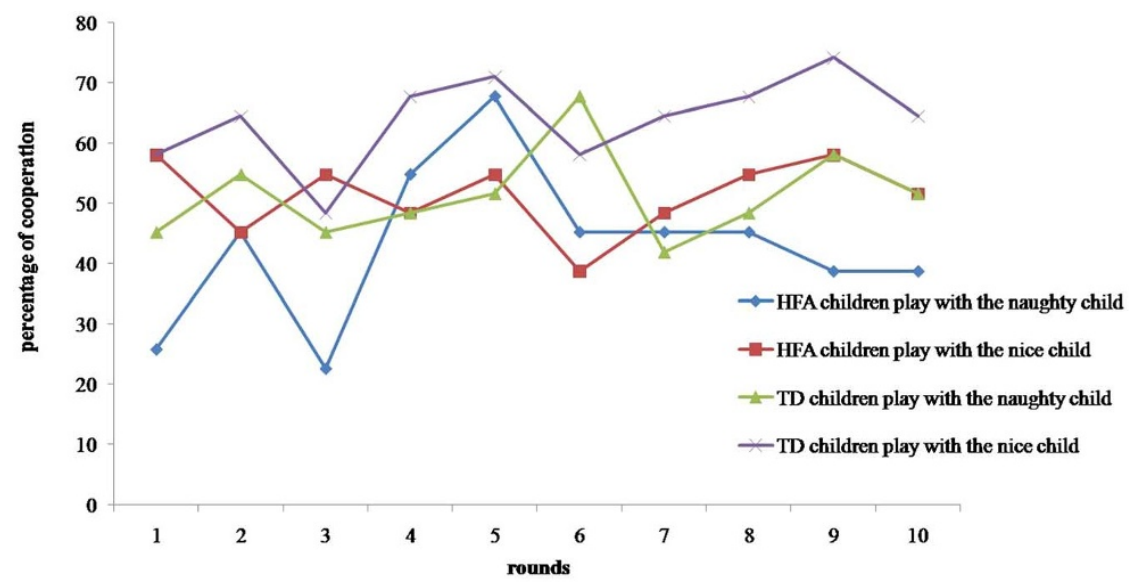

Figure $3 \mid$ described the percentage of choosing cooperative responses when HFA and TD children played with nice child and naughty child across the 10 rounds of the PDG.

children and TD children could make moral judgment correctly in this study, consistent withLeslie, et al. ${ }^{13}$. Thus, following these authors and others(e.g., Grant, et al. ${ }^{19}$ ), HFA children seemed to have little difficulty in evaluating certain acts (such as hitting and sharing) in terms of their morality. On the contrary, HFA children judged harming others as significantly worse than TD children. This indicates that HFA children might have more rigid criteria for what constitutes morally naughty actions. This might be because HFA children are more rule-oriented when it comes to certain behavior because of their disorder. For example, stereotypy, compulsive behavior, sameness, ritualistic behavior, repetitive or restricted behavior have been associated as part of the diagnosis of autism ${ }^{27}$. Thus, HFA children might also be more rule-oriented when it comes to moral actions. Similarly, Baron-Cohen ${ }^{28}$ argued that although autistic individuals are typically self-focused, they are highly moral people, have a strong sense of justice, and think deeply about how to be good.

While HFA children can correctly judge the morality of nice and naughty acts, being partnered with persons of different morality did not change their level of cooperation. Furthermore, HFA children's cooperation was not different when they played with a random stranger, compared with when they played with the nice child or with the naughty child. On the other hand, TD children cooperated more when they played with the nice child than that when they played with the naughty child or the random stranger. These latter findings are in line with previous research ${ }^{22,23}$ which shows that, beginning in the preschool years, TD children take into account their interaction partners' previous moral behavior when deciding whether to act prosocially.

HFA children essentially focus on their own self, and have lower empathic abilities than normally developing children ${ }^{3}$. While some HFA children show empathy with others and overcome their selffocus, this takes great cognitive effort ${ }^{28}$. Being less interested in others and the world outside their own might lead to HFA paying little attention to partner's morality when they play in the PDG, even they had an idea about the morality of the partner. Thus, HFA children's cooperative performance was not influenced by partner's morality, although they could correctly judge others' morality in basic moral judgment stories.

In addition, differences in peer experience between HFA children and TD children might also contribute to finding that TD children show different levels of cooperation with different partners while HFA children do not. HFA children have difficulties in social initiation and social-emotional understanding, but are not insensitive to social stimuli, as they were as likely to interact socially with peers ${ }^{29}$. Autistic children are in a vicious circle of social isolation. On the one hand, they want to interact with peers and to express the feelings of disappointment and loneliness in the absence of interaction. On the other hand, they do not know how to properly interact with peers due to their limited capacity and experience of social and emotional understanding ${ }^{30}$. Children with autism have poor experiences in interacting with peer group in daily life, which might make HFA children show similar levels of cooperation in experimental situations (such as the prisoner's dilemma game) when they played with partner of different morality. In contrast, TD children make many friends and accumulate rich experiences to get along with peers in elementary school, at which stage it is important to develop friendships $^{31}$. Moreover, attention to moral principles, such as norm and promise $^{32}$, becomes an important feature of friendships and peer relations $s^{33}$. Therefore, TD children might be more likely to take into account their partners' characters, including their morality, compared with HFA children.

Furthermore, HFA children also have deficits in reciprocal peer interaction and social cognition. They perform more ritualized behavior and less social interactive behavior (such as prosocial behavior). Moreover, the social interactive behavior performed by autistic children is only to maintain similarity but not to share emotion and experience with peers ${ }^{29}$. Peer interactions provide opportunities to initiate and maintain prosocial behavior with individuals of similar power and status. In these relationships children learn the principles of reciprocity and open communication ${ }^{34}$. Children develop a deep understanding about moral identity by thinking about moral events from different perspectives. Moreover, peers are able to provide warm and powerful resources, which is an important ingredient of prosocial behavior. Peers also give feedback by providing reward and punishment to promote and diminish moral and prosocial behavior. Peers and the experiences based on interacting with peers are important to children's prosocial behavior, trust and intimacy, which are produced through reciprocal prosocial behavior and, are the foundation of the development of positive morals ${ }^{34}$. Peers and peer relationship are important to the development of children's prosocial behavior $^{35,36}$. HFA children's deficiency in peer relationship might lead them to perform indiscriminate cooperation when playing with the naughty and the nice partner in the current study.

In addition, HFA children have typically deficits in social function, based on their impairments in ToM and empathy, although they have normal IQ. Empathy is important for children's development of moral judgment, prosocial behaviors, and social competence ${ }^{37}$. The strong relationship between moral judgment and ToM is also confirmed by neuroimaging evidence ${ }^{10,38,39}$. Furthermore, the relationship between theory of mind and cooperation has also been shown through behavioral evidence ${ }^{40}$ and neuroimaging ${ }^{41-43}$. Thus, HFA children's deficits in social functioning might lead them to 


\begin{tabular}{|c|c|c|c|}
\hline \multirow{2}{*}{$\frac{\text { Cards }}{\text { Children A }}$} & \multicolumn{2}{|c|}{ Payoffs } & \multirow[b]{2}{*}{ Children B } \\
\hline & Children B & Children A & \\
\hline$\overline{0}$ & 0 & 3 & 3 \\
\hline$\Delta$ & $\Delta$ & 2 & 2 \\
\hline$\Delta$ & 0 & 4 & 1 \\
\hline 0 & $\Delta$ & 1 & 4 \\
\hline
\end{tabular}

perform similar cooperation when they interacted with partners of different moralities in prisoner's dilemma game, although they could judge other's morality correctly.

Some limitations of the current study should be acknowledged. Firstly, while HFA and TD children were matched on age, gender, and IQ; differences in children's verbal ability were not controlled for. Future research should measure HFA children's language ability before examining their social behavior. Secondly, although the autistic children in this study were evaluated by the expert clinician based on DSM-IV criteria and their diagnosis was confirmed by other multiple clinical evaluation (see details in Method section), their diagnosis was not confirmed by the Autism Diagnostic Observation Schedule (ADOS). Future research should use this more standardized clinical instrument to ensure a research-quality diagnosis. In addition, more sophisticated moral judgments should be used further in the future. For example, since HFA children might have particular difficulties with understanding others' intentions moral judgments based on others' intentions and cooperation with well- and ill-intended partners might be an interesting direction for future studies.

Overall, this study found that both HFA children and TD children could make correct moral judgments, and HFA children might have even more rigid criteria for what constitutes a "naughty" act than TD children. HFA children's cooperation was similar when they played with partners of different moralities, while TD children showed higher cooperation when they played with a morally nice child than that when they interacted with a naughty child. Therefore, HFA children's cooperation was not influenced by partner's morality, while TD children's cooperation might be prompted by partner's nice morality. This study thus gives an important insight into high-functioning autistic children's moral judgment and moral behavior.

\section{Methods}

Participants. Thirty-eight children with autism aged between 6 to 12 years $\left(M_{\text {Age }}=\right.$ $9.59, S D=2.29,30$ boys and 8 girls) were recruited. The gender ratio was nearly $4: 1$, which is similar to previous research ${ }^{44}$. All of the participants were diagnosed by professional hospitals according to the autism diagnostic criteria specified in the DSM-IV. All participants' performance on the Autism Behavior Checklist (ABC) and Childhood Autism Rating Scale (CARS) reached the cut-off score for autism. Moreover, HFA children's performance on the Autism Spectrum Quotient Children's Version ${ }^{45}$ also reached the cut-off score of 76. Participants' IQ was tested with the standardized Raven test ${ }^{46}$. Autistic children's IQ was between 70 and 144 with an average of 110, indicating that they were HFA children.

As only 31 of the 38 HFA children completed the prisoner's dilemma game (PDG, see below), 31 TD children were also recruited, who were matched to the HFA children based on their age and gender. TD children's IQ was also tested using the standardized Raven test and ranged between 83 and 130, with an average of 113 There was no significant difference between the two groups of children in terms of IQ, $t(67)=-0.774, p=0.442$.

Materials and procedure. Moral judgment task. Each child was told two moral stories $^{13}$, and was asked whether the protagonist in the story was a nice or naughty child. Participants were introduced to a response scale and were trained to use it before formal testing. The response scale showed three stars at one end of a line, and three Xs at the other end. Children were told that the stars meant that something was "really nice," and the Xs meant that something was "really naughty." A black circle in the middle of the scale indicated that something was "just ok." A single star and a single X, which meant "a little nice" and "a little naughty", respectively, were at points in between the midpoint and the two extremes. After the scale was explained to them, children were asked to show how much they liked various items, such as ice cream, spinach, and water, by pointing to the appropriate point on the scale.

Children were told both the "good" and "naughty" moral story in counterbalanced order. For the naughty story, children were first introduced to the story protagonists (illustrated with dolls) and then told the harm story:

"Today teacher Wang's class has an exciting activity. Everyone gets to pick a special toy. Lele fortunately gets to pick a toy first, and he/she picks a stuffed monkey. It makes Mingming angry, because Mingming also wants to have the stuffed monkey. Mingming hits Lele in the arm and this makes Lele very sad, and Lele starts to cry".

Children were then asked two questions:

Nice/naughty question: "Was it nice, naughty, or just okay that Mingming hit Lele?"

Response scale: "Show me how nice or naughty it was on the Scale".

In the nice condition, children were also first introduced to the story protagonists (illustrated with dolls), and then they were told the kindness story:

“Today Miss Wang's class is eating. Lele has no candy. This makes Lele very sad and Lele starts to cry. This is Junjun, and Junjun has two pieces of candy. Junjun shares his/her candy with Lele. This makes Lele very happy, and Lele starts to laugh".

Children were then asked two questions:

Nice/naughty question: "Was it nice, naughty, or just okay that Junjun shares candy with Lele?"

Response scale: "Show me how nice or naughty it was on the Scale."

Cooperative task. The classic prisoner's dilemma game (PDG) was adopted to investigate children's cooperative behavior. There were 10 rounds in all in each condition. To make sure that HFA children were able to understand the rules of game, the matrix of payoffs in PDG was simplified in this study, as shown in Table 2.The shape $\bigcirc$ was adopted to represent the choice for cooperation, while the shape $\Delta$ represented the choice for competition. Geometric shapes were chosen to avoid the influence of the semantic meaning of the words "cooperation" and "competition" for HFA and TD children. Children were asked to play the game with a random stranger, who was the experimenter's confederate.

Participants were first introduced to the two cards, $\bigcirc$ and $\Delta$, and were told that s/ he and the partner needed to freely select one of the cards in each round and show the selected card to each other simultaneously after hearing a sound signal. Then the experimenter explained the payoff of choices to children and emphasized that their payoff was determined by the choice of both sides. Children were also asked to record their own and partner's choices and payoffs on paper after each round so they could get feedback and understand their choices deeply. Child-friendly language was used to make children, especially autistic children, understand how to play the games.

To make sure that children with autism had understood the rules of game, a practice trial was conducted before the actual game. Children were asked which payoffs they could get after making certain choices. The actual game only started after they correctly answered this payoff question for three times in a row. If they could not answer correctly, the rules of the game were repeated. If after three times, they still could not pass the practice questions, the game was stopped. The total payoffs children and the partner got were calculated at the end of each condition. The partner always adopted the tit-for-tat strategy: $S /$ he chose to cooperate in the first round of the game, but in the following rounds chose the strategy that had been adopted by the partner in the preceding round. After playing all the games, children were given gifts as real payoff.

After children judged protagonists' morality correctly in the moral judgment tasks, they were asked to interact with three protagonists (a nice child and a naughty child from the story) in PDG in counterbalanced order. Only children who could correctly judge the protagonists' morality in the moral stories were asked to interact with a morally nice or naughty protagonist in PDG. In addition, all children were asked to play PDG with a random stranger, the experimenter's confederate, whose morality was not known to participants. Children's cooperative behavior in this neutral con dition was used as a baseline. In each condition, children interacted with the same partner (nice child, naughty child, or the random stranger) for ten times.

The ethics committee in Institute of Psychology, Chinese Academy of Sciences, had approved our experiments. Informed consent forms for children's parents had been obtained from all subjects and methods were carried out in accordance with the approved guidelines.

1. Baron-Cohen, S. \& Wheelwright, S. The empathy quotient: an investigation of adults with Asperger syndrome or high functioning autism, and normal sex differences. J. Autism Dev. Disord. 34, 163-175 (2004).

2. Frith, U. Autism: Explaining the enigma. Br. J. Dev. Psychol. 21, 465-468 (2003).

3. Kanner, L. Autistic disturbances of affective contact. Nervous child 2, 217-250 (1943).

4. Kasari, C., Chamberlain, B. \& Bauminger, N. in Development and Autism: Perspectives from Theory and Research (eds Burack, J., Charman, T., Yirmiya, N. \& Zelazo, P.) 309-323 (Erlbaum Press, 2001).

5. Blair, R. J. R. Responding to the emotions of others: Dissociating forms of empathy through the study of typical and psychiatric populations. Conscious. Cogn. 14, 698-718 (2005).

6. Baron-Cohen, S. Mindblindness: As essay on autism and theory of mind. (Cambridge, MA: MIT Press, 1995).

7. Golan, O., Baron-Cohen, S., Hill, J. J. \& Rutherford, M. The 'reading the mind in the voice' test-revised: A study of complex emotion recognition in adults with and 
without autism spectrum conditions. J. Autism Dev. Disord. 37, 1096-1106 (2007).

8. Downs, A. \& Smith, T. Emotional understanding, cooperation, and social behavior in high-functioning children with autism. J. Autism Dev. Disord. 34, 625-635 (2004).

9. Kahn, P. H. Mind and morality. New Dir. Child Adolesc. Dev. 2004, 73-83 (2004).

10. Young, L., Cushman, F., Hauser, M. \& Saxe, R. The neural basis of the interaction between theory of mind and moral judgment. Proc. Natl. Acad. Sci. 104 8235-8240 (2007).

11. García, A. E. V. \& Ostrosky-Solís, F. From morality to moral emotions. Int. J. Psychol. 41, 348-354 (2006).

12. Krahn, T. \& Fenton, A. Autism, empathy and questions of Moral Agency. J. Theory Soc. Behav. 39, 145-166 (2009).

13. Leslie, A. M., Mallon, R. \& Dicorcia, J. A. Transgressors, victims, and cry babies: Is basic moral judgment spared in autism? Soc. Neurosci. 1, 270-283, doi:10.1080/ 17470910600992197 (2006).

14. Moran, J. M. et al. Impaired theory of mind for moral judgment in highfunctioning autism. Proc. Natl. Acad. Sci. U. S. A. 108, 2688-2692 (2011).

15. Killen, M., Lee-Kim, J., McGlothlin, H. \& Stangor, C. How children and adolescents evaluate gender and racial exclusion. Monogr. Soc. Res. Child Dev. 67, 1-129 (2002).

16. Smetana, J. G. in Handbook of moral development (eds Killen, M. \& Smetana, J. G.) 119-154 (NJ: Erlbaum, 2006)

17. Blair, R. J. R. Brief report: Morality in the autistic child. J. Autism Dev. Disord. 26 571-579 (1996).

18. Feng, Y. \& Su, Y. J. The Moral and Conventional Judgment in Autistic Children. Chin. J. Spec. Educ. 6, 65-69 (2005).

19. Grant, C. M., Boucher, J., Riggs, K. J. \& Grayson, A. Moral understanding in children with autism. Autism 9, 317-331 (2005).

20. Eisenberg-Berg, N. \& Hand, M. The relationship of preschoolers' reasoning about prosocial moral conflicts to prosocial behavior. Child Dev. 50, 356-363 (1979).

21. Janssens, J. M. \& Dekovic, M. Child rearing, prosocial moral reasoning, and prosocial behaviour. Int. J. Behav. Dev. 20, 509-527 (1997).

22. Olson, K. R. \& Spelke, E. S. Foundations of cooperation in young children. Cognition 108, 222-231 (2008)

23. Kenward, B. \& Dahl, M. Preschoolers distribute scarce resources according to the moral valence of recipients' previous actions. DP 47, 1054 (2011).

24. Killen, M. in Handbook of moral behavior and development (eds Kurtines, W. M. \& Gerwirtz, J. L.) 115-138 (Lawrence Erlbaum Associates, Inc., 1991).

25. Sally, D. Into the looking glass: discerning the social mind through the mindblind. Adv. Group Processes 18, 99-128 (2001).

26. Auyeung, B. et al. The children's empathy quotient and systemizing quotient: Sex differences in typical development and in autism spectrum conditions. J. Autism Dev. Disord. 39, 1509-1521 (2009).

27. Lam, K. S. \& Aman, M. G. The Repetitive Behavior Scale-Revised: Independent validation in individuals with autism spectrum disorders. J. Autism Dev. Disord. 37, 855-866 (2007).

28. Baron-Cohen, S. in The Lost Self: Pathologies of Brain and Identity (eds Todd E. Feinberg \& Julian Paul Keenan) 166-180 (Oxford University Press, 2005).

29. Sigman, M. \& Ruskin, E. Continuity and change in the social competence of children with autism, Downs syndrome, and developmental delays. Monogr. Soc. Res. Child Dev. 64, 109-113 (1999).

30. Bauminger, N. \& Kasari, C. Loneliness and Friendship in High-Functioning Children with Autism. Child Dev. 71, 447-456 (2000).

31. Hartup, W. W. in Handbook of child psychology Vol. 4 (eds Mussen, P. H. (Series Ed.) \& Hetherington, E. M. (Vol. Ed.)) 103-196 (Wiley, 1983).

32. Kohlberg, L. Essays on moral development. Vol. 2, The psychology of moral development: the nature and validity of moral stages. Vol. 2 (Harper \& Row, 1984).

33. Bigelow, B. J. Children's friendship expectations: A cognitive-developmental study. Child Dev., 246-253 (1977).
34. Carlo, G. Care-based and altruistically-based morality. Handbook of moral development, 551-579 (2006)

35. Eisenberg, N. \& Fabes, R. A. in Handbook of child psychology (5th) Vol. 3 (ed Damon, W.) 701-778 (Wiley \& Sons, 1998).

36. Wentzel, K. R. \& McNamara, C. C. Interpersonal relationships, emotional distress, and prosocial behavior in middle school. J. Early Adolesc. 19, 114-125 (1999)

37. Soenens, B., Duriez, B., Vansteenkiste, M. \& Goossens, L. The intergenerational transmission of empathy-related responding in adolescence: The role of maternal support. Pers. Soc. Psychol. B. 33, 299-311 (2007).

38. Young, L., Camprodon, J. A., Hauser, M., Pascual-Leone, A. \& Saxe, R. Disruption of the right temporoparietal junction with transcranial magnetic stimulation reduces the role of beliefs in moral judgments. Proc. Natl. Acad. Sci. 107, 6753-6758 (2010)

39. Young, L. \& Saxe, R. Innocent intentions: A correlation between forgiveness for accidental harm and neural activity. Neuropsychologia 47, 2065-2072 (2009).

40. Paal, T. \& Bereukei, T. Adult theory of mind, cooperation, Machiavellianism: The effect of mindreading on social relations. Pers. Individ. Dif. 43, 541-551 (2007).

41. Lissek, S. et al. Cooperation and Deception Recruit Different Subsets of the Theory-of-Mind Network. PLoS One 3, e2023, doi:10.1371/journal.pone.0002023 (2008)

42. Mitchell, J. P., Banaji, M. R. \& MacRae, C. N. The link between social cognition and self-referential thought in the medial prefrontal cortex. J. Cognit. Neurosci. 17, 1306-1315 (2005).

43. Rilling, J. K., Sanfey, A. G., Aronson, J. A., Nystrom, L. E. \& Cohen, J. D. The neura correlates of theory of mind within interpersonal interactions. NeuroImage 22, 1694-1703 (2004)

44. Wing, L. Asperger's syndrome: A clinical account. Psychol. Med. 11, 115-129 (1981).

45. Auyeung, B., Baron-Cohen, S., Wheelwright, S. \& Allison, C. The autism spectrum quotient: Children's version (AQ-Child). J. Autism Dev. Disord. 38, 1230-1240 (2008).

46. Wang, D. \& Qian, M. Combined Raven's test (second revision in Chinese). (Department of Medical Psychology in Tianjin Medical University, 1997).

\section{Acknowledgments}

This project was supported by the National Nature Science Foundation of China [31300859, 30970911], the Scientific Foundation of Institute of Psychology, Chinese Academy of Sciences [Y2CQ022005], China 973 Program [2010CB8339004] and Chinese Academy of Sciences project [KJZD-EW-L04].

\section{Author contributions}

J.L. and L.Z. designed the experiment. J.L. collected and analyzed the data. J.L., L.Z. and M.G. wrote and reviewed the manuscript.

\section{Additional information}

Competing financial interests: The authors declare no competing financial interests.

How to cite this article: Li, J., Zhu, L.Q. \& Gummerum, M. The relationship between moral judgment and cooperation in children with high-functioning autism. Sci. Rep. 4, 4314 DOI:10.1038/srep04314 (2014)

(c) (i) (5) $\odot$ This work is licensed under a Creative Commons Attribution-

By NC ND NonCommercial-NoDerivs 3.0 Unported license. To view a copy of this license, visit http://creativecommons.org/licenses/by-nc-nd/3.0 